\title{
ÁCIDOS GRASOS Y ESTEROLES EN LA CERÁMICAARQUEOLÓGICA DE LOS LAGOS MUSTERS Y COLHUE HUAPI (BAJO SARMIENTO, PROVINCIA DEL CHUBUT)
}

\author{
FATTY ACIDS AND STEROLS IN ARCHAEOLOGICAL POTTERY OF LAKES MUSTERS \\ AND COLHUE HUAPI (BAJO SARMIENTO, CHUBUT PROVINCE)
}

\author{
Verónica Schuster ${ }^{1}$, Marcia Mazzuca $^{2}$, Celeste Gurín $^{3}$ y Eduardo Moreno ${ }^{4}$
}

Recibido 28 mayo 2020. Aceptado 30 noviembre 2020

\begin{abstract}
Resumen: Este trabajo expone los primeros resultados de estudios de ácidos grasos absorbidos en cerámicas arqueológicas de sitios del bajo Sarmiento ubicado en el centro sur de la provincia del Chubut. Se presenta el análisis efectuado en diez muestras cerámicas procedentes de ocho localidades arqueológicas que fueron procesadas a partir del uso combinado de cromatografía gaseosa y espectrometría de masas. Los resultados se discuten teniendo en cuenta los recursos locales y potencialmente disponibles para el procesamiento y/o consumo en dicha tecnología. Además, se enfatiza en las ventajas y limitaciones metodológicas inherentes a la identificación y la determinación de las diversas especies y recursos a partir de muestras de cerámicas arqueológicas.

Palabras clave: Bajo Sarmiento, cerámica arqueológica, ácidos grasos, Patagonia Argentina.
\end{abstract}

\begin{abstract}
This paper presents the first results of studies of fatty acids absorbed in archaeological ceramics low Sarmiento sites located in the Chubut southern - center province. The analysis carried out on ten ceramic samples from eight archaeological sites that were processed from the combined use of gas chromatography and mass spectrometry is presented.

The results are discussed taking into account local resources potentially available for processing and / or consumption such technology. Also, emphasis will be placed on the methodological advantages and limitations inherent in the identification and determination of the various species and resources from samples of archaeological ceramics.

Key words: Sarmiento Basin, archaeological pottery, fatty acids, Patagonia Argentina.
\end{abstract}

\section{Introducción}

El bajo Sarmiento es una depresión de origen tectónicoerosivo formado por laderas relativamente abruptas y un fondo de valle plano, que se conectó con la cuenca del río Senguer formando así un gran cuerpo de agua denominado paleolago Sarmiento (González Díaz \& Di Tommaso, 2014). Dicho fenómeno se vincula con la última desglaciación -es decir durante el límite Pleistoceno-Holoceno- y habría alcanzado un nivel de 60 metros sobre el actual lago Musters. Hacia el Holoceno medio, el paleolago se hallaría unos 30 metros por encima de ese nivel, y ya hacia el Holoceno tardío final, este único cuerpo de agua se desarticularía dando lugar a los lagos Musters y Colhué Huapi, exponiendo así, el fondo actual del valle de Sarmiento (Figura 1; Moreno et al., 2016). Las ocupaciones humanas más antiguas registradas hasta el momento en el área se ubican en las laderas con cronologías entre $c a$. 5000 hasta el 1600 años AP, siendo generalmente ocupaciones breves probablemente basadas en la caza de animales terrestres. Con posterioridad a los 1500 años AP, el fondo del valle también es ocupado, detectándose sitios arqueológicos de mayor tamaño, con una mayor densidad de artefactos y con una fuerte estructuración intrasitio. Específicamente en el perilago del Colhue Huapi se detectó una importante actividad relacionada con la captura de recursos fluviales. En efecto, la información arqueofaunística del sitio Valle Hermoso 4 mostró la prevalencia casi absoluta de peces asociado a una elevada frecuencia de pesos de red (Moreno et al. 2015; Reyes \& Svoboda, 2016); mientras que otros sitios al noroeste del lago Musters -Arroyo Vulcana 1 y Laguna La Flecha 10- manifiestan un substancial consumo de peces aunque en menor proporción respecto del guanaco y otros recursos terrestres menores (Moreno \& Svodoba 2013). Por lo tanto, si bien se observa una importante actividad de pesca en el área de estudio, se aprecia la continuidad del consumo del guanaco, principal recurso de los cazadores recolectores de Patagonia.

Por otro lado en el bajo de Sarmiento es muy frecuente el hallazgo de cerámica tanto de manera aislada como en sitios arqueológicos. Los análisis de estos materiales han comenzado recientemente y están orientados a determinar la procedencia, las características tecnológicas-decorativas y el uso o la función de esta tecnología. Ya se han realizado análisis macroscópicos

1 Instituto de Diversidad y Evolución Austral (IDEAus), Centro Nacional Patagónico (CENPAT) CONICET, Bv. Almte. Brown 2915, U9120 Puerto Madryn, Chubut, Argentina. schuster@cenpat-conicet. gob.ar.

2 Centro de Investigación y Transferencia Golfo San Jorge (CIT GSJ), CONICET, Universidad Nacional de la Patagonia San Juan Bosco (UNPSJB), Km 4, 9000 Comodoro Rivadavia, Chubut, Argentina. mazzucam@unpata.edu.ar.

3 Departamento de Química, Facultad de Ciencias Naturales y Ciencias de la Salud Universidad Nacional de la Patagonia San Juan Bosco (UNPSJB), Km 4, 9000 Comodoro Rivadavia, Chubut, Argentina.

4 Departamento de Historia, Facultad de Humanidades y Ciencias Sociales, Universidad Nacional de la Patagonia San Juan Bosco (UNPSJB), Km. 4, 9000 Comodoro Rivadavia, Chubut, Argentina. 
de todo el material cerámico registrado hasta el momento con el objetivo de evaluar las tendencias tecno-morfológicas. A partir de dicha tarea, se ha identificado que este conjunto cerámico comparte algunas características generales con otros grupos cerámicos de Patagonia, como por ejemplo las formas globulares o subglobulares, la ausencia de bases formatizadas y asas o apéndices y la baja frecuencia de piezas pintadas. No obstante, en este caso en particular hay una importante cantidad y variedad de tiestos decorados. Los estudios estilísticos y decorativos emprendidos a la fecha permitieron identificar técnicas y motivos no frecuentes para la región patagónica. Asimismo, se han llevado a cabo estudios petrográficos de las pastas para explorar la procedencia de la materia prima. Los primeros resultados permiten proponer que la mayor parte de la cerámica analizada correspondería a piezas elaboradas en el área ya que la composición mineralógica resulta compatible con la geología local, si bien se espera complementarla a corto plazo con otras técnicas como la difracción de rayos $\mathrm{X}$ y el análisis de diatomeas.

La mayor frecuencia de fragmentos cerámicos se encuentra en el fondo del valle, por lo que de acuerdo con la reconstrucción del paleolago, serían posteriores al 1500 años AP y resultaría concordante con la presencia de la tecnología cerámica en la región central de Patagonia (Schuster, 2014). La coincidencia cronológica entre el uso de cerámica y la actividad de pesca por parte de los cazadores recolectores del bajo Sarmiento, sugiere al menos inicialmente, la posibilidad de que ambos fenómenos estén relacionados. Por ello, se propuso como hipótesis inicial que los contenedores cerámicos hayan sido una tecnología vinculada con el procesamiento y consumo de peces en esta región en particular.

En este sentido, el objetivo en curso pretende obtener información acerca del uso de la tecnología cerámica a través del análisis de ácidos grasos y esteroles que podrían haber quedado absorbidos en la matriz de los fragmentos cerámicos recuperados en la zona de estudio.

En la Patagonia Argentina se han realizado algunos estudios basados en la caracterización química de residuos de lípidos absorbidos en cerámica arqueológica (Chaile et al., 2018; Cordero \& March, 2013; Gómez Otero et al., 2014; Schuster, 2014; Stoessel et al., 2015) pudiéndose atribuir diversos usos a las piezas cerámicas, así como también, identificar diferentes tipos de recursos vegetales y animales (tanto sean terrestres, marinos y/o dulceacuícolas).

El presente trabajo es el primer análisis químico efectuado en cerámicas del bajo de Sarmiento a efectos de verificar la presencia de lípidos, y a partir de ello, las características de los perfiles de ácidos grasos y esteroles. Actualmente, se están confirmando algunos datos cromatográficos y realizando identificaciones de otras moléculas presentes en las muestras por espectrometría de masas, por lo que los resultados aquí expuestos deben tomarse como un estudio preliminar.

\section{Materiales}

La totalidad de las muestras cerámicas seleccionadas proceden de superficie, por lo que han estado expuestas naturalmente a las adversidades climáticas. Además, debido a su contexto de recuperación superficial no contamos con fechados directos ni otros hallazgos arqueológicos asociados. En general estos materiales se registraron sobre depósitos eólicos, a excepción de los fragmentos E269, E270 y E271 que se hallaron en suelos hidromórficos, es decir, en superficies que periódicamente han estado inundadas y por ello tienen alto contenido orgánico. La muestra E274, es la única que se recuperó sobre un depósito de un antiguo fandelta.

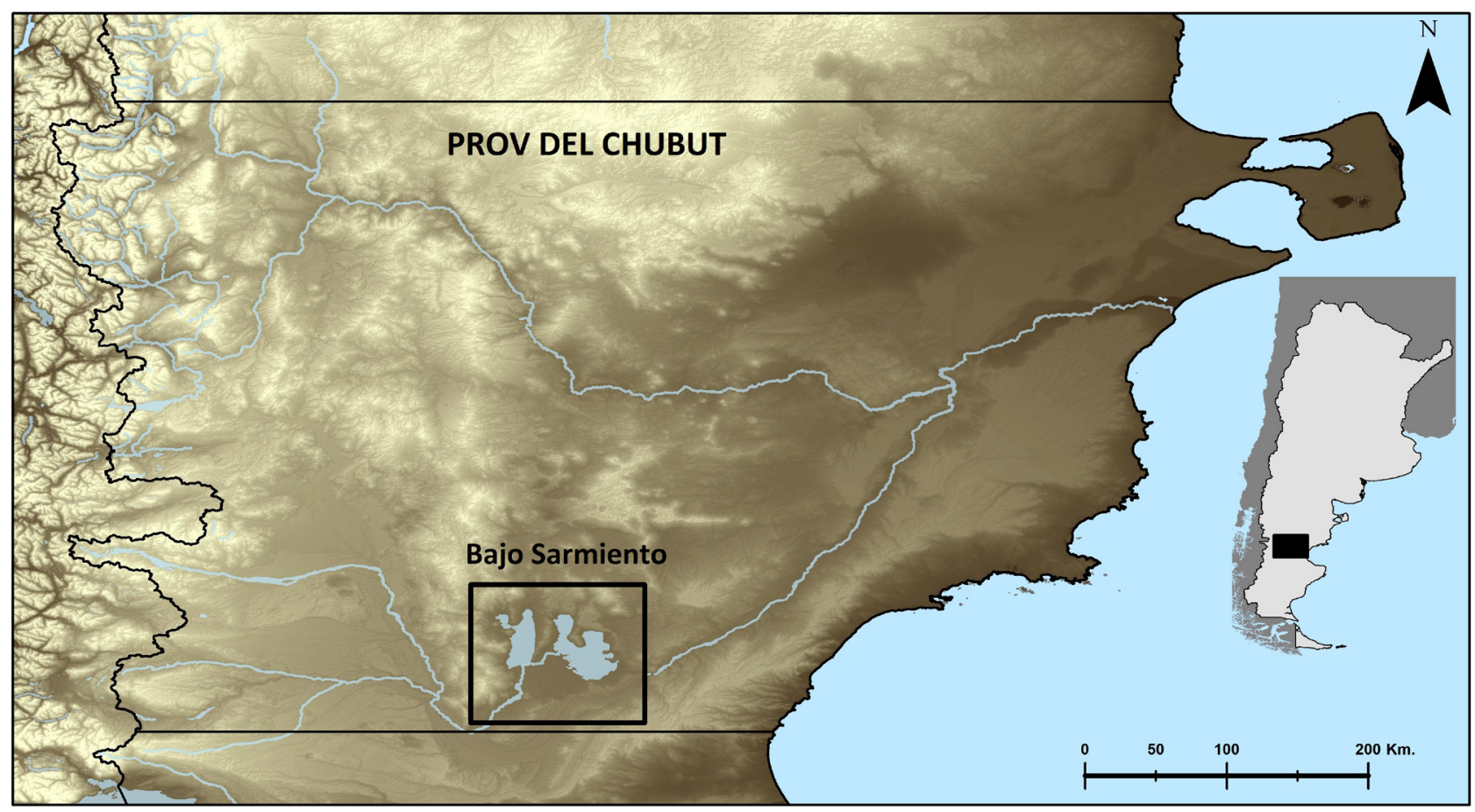

Figura 1. Área de estudio: bajo Sarmiento en la provincia del Chubut, Patagonia Argentina. 
Tabla 1. Sitios arqueológicos, ubicación geográfica y características topográficas.

\begin{tabular}{|c|c|c|c|}
\hline $\mathbf{N}^{\mathbf{0}}$ Muestra & Sitio & Ubicación & Topografía \\
\hline E265 & Península Baya & $\begin{array}{c}\text { Norte del Lago } \\
\text { Muster }\end{array}$ & Fondo de valle \\
\hline E266 & Valle Hermoso 3 & $\begin{array}{c}\text { Sudeste del lago } \\
\text { Colhué Huapi }\end{array}$ & Fondo de valle \\
\hline E267 & Codo del Senguer 1 & Valle del río Senguer & Valle fluvial \\
\hline E268 & El Fortín 32 & Este del lago Muster & Fondo de valle \\
\hline E269 & Boliche Jerez 18 & $\begin{array}{c}\text { Noroeste del lago } \\
\text { Colhué Huapi }\end{array}$ & Fondo de valle \\
\hline E270 & La Campanita & $\begin{array}{c}\text { Sudeste del lago } \\
\text { Colhué Huapi }\end{array}$ & Fondo de valle \\
\hline E271 & Boliche Jerez 18 b & $\begin{array}{c}\text { Noroeste del lago } \\
\text { Colhué Huapi }\end{array}$ & Fondo de valle \\
\hline E272 & Valle Hermoso 4 & $\begin{array}{c}\text { Sudeste del lago } \\
\text { Colhué Huapi }\end{array}$ & Fondo de valle \\
\hline E273 & Cañadón Bóer Alto 3 & $\begin{array}{c}\text { Norte del lago } \\
\text { Colhué Huapi }\end{array}$ & Ladera \\
\hline E274 & $\begin{array}{c}\text { Delta del Arroyo } \\
\text { Vulcana }\end{array}$ & $\begin{array}{c}\text { Noreste del lago } \\
\text { Muster }\end{array}$ & Fondo de valle \\
\hline
\end{tabular}

Para este trabajo del total de los materiales cerámicos disponibles se seleccionaron 10 fragmentos cerámicos procedentes de ocho sitios arqueológicos con emplazamientos topográficos diversos respecto a las cuencas de los lagos de la región en estudio (Figura 2, Tabla 1). Si bien se intentó maximizar la diversidad de procedencia de las muestras, la gran mayoría de la cerámica procede de sitios del fondo del valle, lo que de acuerdo al modelo del paleolago mencionado párrafos arriba, correspondería al Holoceno Tardío Final, es decir a momentos posteriores a los 1500 años AP.
Para la elección de las muestras se consideraron fragmentos de un tamaño promedio de $2 \mathrm{~cm} 2$ y dentro de lo posible, que correspondieran a la sección media del cuerpo, si bien con excepción de dos fragmentos -E268 y E273- se desconoce la ubicación de cada fragmento respecto de la pieza original (Tabla 2). En general, estas cerámicas revelan un tratamiento de superficie alisada -aunque algunas caras internas están exfoliadas (Tabla 2), no presentando residuos adheridos ni carbonizaciones visibles macroscópicamente. Finalmente, cinco de estos tiestos contaban con estudios petrográficos previos, y tal como se

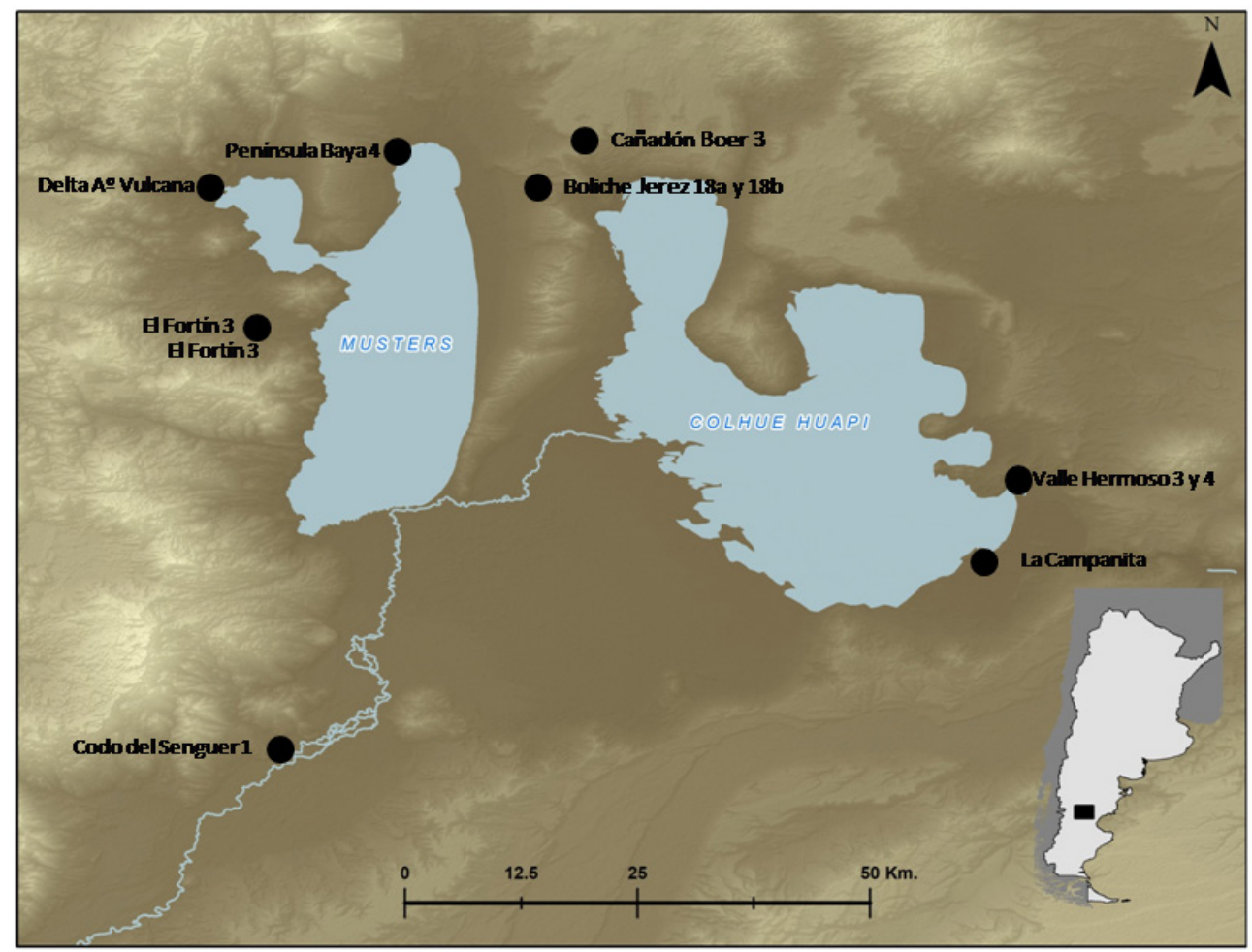

Figura 2. Ubicación de los sitios arqueológicos considerados en este trabajo. 


\begin{tabular}{|c|c|c|c|c|c|}
\hline No Muestra & Sitio & Procedencia & Espes or* & Segmento & $\begin{array}{c}\text { Tratamiento } \\
\text { superficie }\end{array}$ \\
\hline E265 & Península Baya & Local & 6.34 & Indet. & Exfoliado I \\
\hline E266 & Valle Hermoso 3 & $?$ & 6.01 & Indet. & Alisado I/E \\
\hline E267 & Codo del Senguer 1 & Local & 5.35 & Indet. & Pulido I/E \\
\hline E268 & E1 Fortín 32 & $?$ & 6.78 & Cuerpo & Alisado I/E \\
\hline E269 & Boliche Jerez 18 & $?$ & 5.10 & Indet. & Alisado I/E \\
\hline E270 & La Campanita & $?$ & 5.48 & Indet. & Alisado I/E \\
\hline E271 & Boliche Jerez 18 b & $?$ & 7.05 & Indet. & Exfoliado I \\
\hline E272 & Valle Hermoso 4 & Local & 5.18 & Indet. & Exfoliado I \\
\hline & & & & & Alisado I/E \\
\hline E273 & Cañadón Bóer Alto 3 & Local & 6.81 & Cuerpo & Hollín sup.I. \\
\hline E274 & $\begin{array}{c}\text { Delta del Arroyo } \\
\text { Vulcana }\end{array}$ & Alóctono? & 5.35 & Indet. & Alisado I/E \\
\hline
\end{tabular}

Tabla 2. Características macroscópicas generales de los fragmentos seleccionados para análisis Referencias: Indet. (indeterminado), I (interno), E (externo), (* promedio).

indica en la Tabla 2, estas cerámicas han sido consideradas de manufactura local.

\section{Metodología}

Las muestras cerámicas seleccionadas para análisis fueron limpiadas mecánicamente con un cepillo de cerdas suaves y trasladadas hacia el laboratorio de Química Orgánica del Departamento de Química de la Universidad Nacional de la Patagonia San Juan Bosco para su extracción y posterior derivatización. La metodología de trabajo se resume en la Figura 3 .

El estudio de ácidos grasos fue realizado acorde a la propuesta de Maier et al. (2007), que consiste en una saponificación y posterior conversión de los ácidos grasos en sus ésteres metílicos de ácidos grasos (FAME) para el análisis cromatográfico. Por su parte, la extracción, purificación e identificación de esteroles fue realizado según lo descripto por Lantos et al. (2015) mediante su transformación de trimetilsilil derivados (TMS).

Una vez procesadas las muestras, fueron enviadas al laboratorio de Cromatografía y Química Orgánica, CENPAT, CONICET, para su análisis cromatográfico. El mismo fue realizado en un cromatógrafo de gases marca Thermo Scientific FOCUS/ISQ con detector de masa simple cuadrupolo Thermo ISQ.

La identificación de los compuestos fue realizada mediante comparación de los tiempos de retención y sus fragmentaciones. Se utilizaron patrones de FAMES Supelco CRM4788, de fitoesteroles vegetales (estigmasterol, campesterol y sitoesterol) (Fluka, Sigma Aldrich) y de colesterol (Sigma Aldrich) como referencia. La abundancia relativa de cada FAME fue calculada en base a las áreas de los picos del cromatograma de iones totales. Se realizó un solo análisis por muestra debido a la escasez del material

\section{Resultados y Discusión}

Como se ha mencionado anteriormente, los materiales cerámicos analizados en este trabajo fueron hallados en superficie, por lo que han estado expuestos naturalmente al oxígeno y a las características climáticas de la Patagonia tales como la erosión del viento y el agua. Además, los sitios arqueológicos de donde provienen las muestras E-269 y E-271, se emplazan sobre suelos hidromórficos, lo que sugiere que han estado sumergidos durante los períodos de ascenso en los niveles de agua de la superficie de los lagos. Similar situación pudo haber ocurrido con las muestras E266 y E 270 dado que se encuentran en suelos muy próximos a estos sitios, y además, la muestra E274 se recolectó sobre un delta antiguo. Una característica observada fue que algunas caras internas estaban exfoliadas, y particularmente la muestra E273, presentaba hollín en la superficie interna. Las condiciones anóxicas así como las fuentes externas de calor (fogones) pueden alterar o condicionar la preservación de los lípidos y los ácidos grasos. No obstante, el primer resultado importante de este trabajo fue que la totalidad de las muestras cerámicas contenían material lipídico.

El análisis cromatográfico de los FAME (Tabla 3), permitió identificar entre 12 a 15 ácidos grasos saturados e insaturados, con presencia y proporción variable. En ninguna de las muestras se identificaron ácidos grasos de menos de 14 átomos de carbono. Esto ocurre en general porque los ácidos grasos de cadena corta son más suceptibles a la hidrólisis, tienden a ser más volátiles y solubles en agua, y se pierden más rápidamente (Evershed 2002). Por otro lado, a excepción de la muestra E-270, en ninguna de las restantes muestras se identificaron ácidos grasos de más de 18 átomos de carbono. En general, los ácidos grasos de cadena muy larga, así como los de cadena menor a 14 átomos de C se deterioran más rápido que los de longitud media (14 a 18 átomos de C). Aunque los ácidos grasos sean más resistentes a la descomposición comparados con otras moléculas, la degradación ocurre cuando están expuestos a factores tales como el oxígeno y 


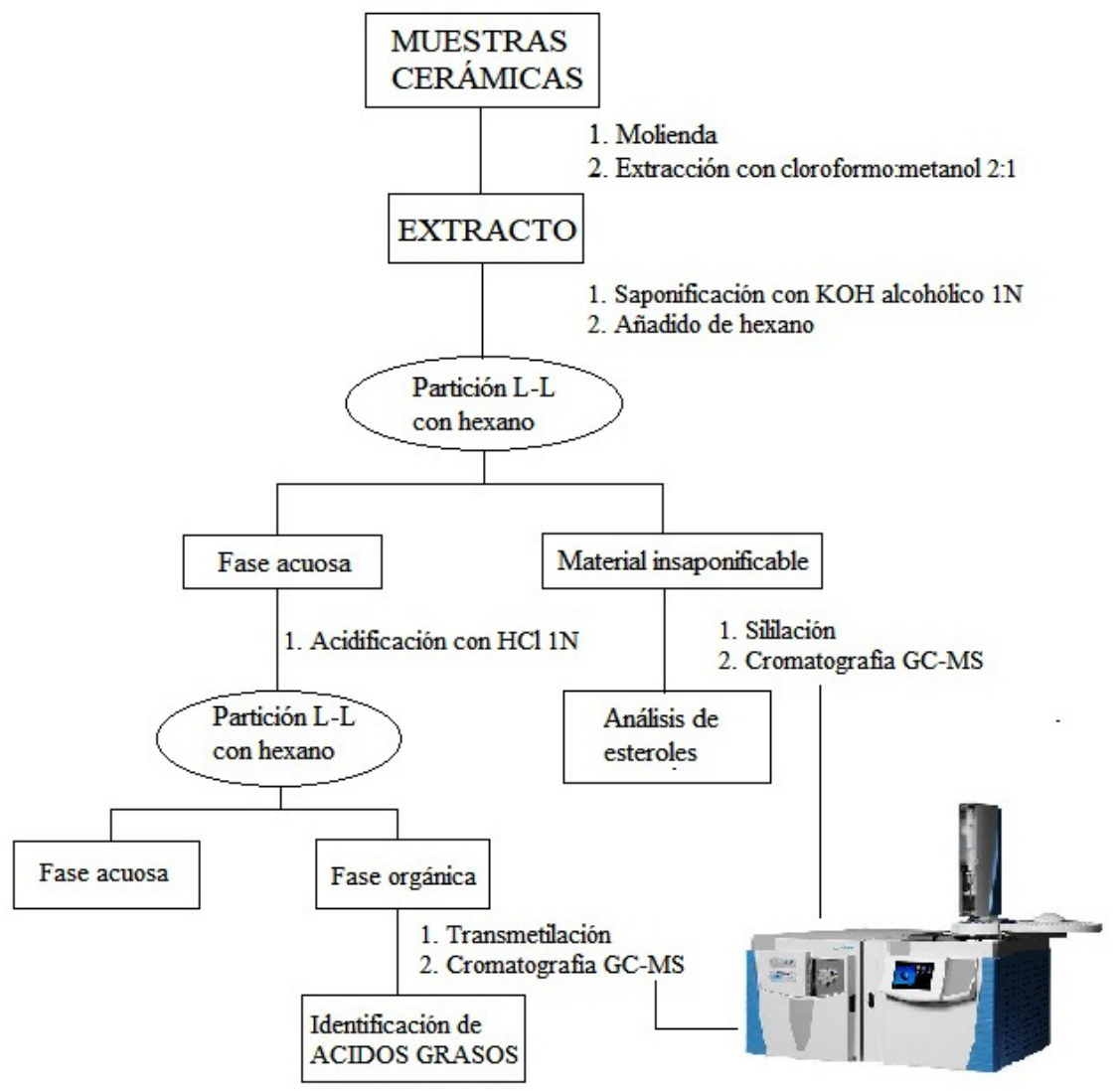

Figura 3. Esquema de trabajo en el laboratorio.

el agua (Eerkens et al., 2007).

En todas las muestras se identificaron ácidos grasos insaturados palmitoleico 16:1 y oleico 18:1, que se encuentran en proporción relativamente elevada junto al ácido linoleico 18:2. Este hecho denotaría un buen grado de conservación de los residuos en sus matrices cerámicas (Evershed et al., 1997; Maier et al., 2007). La preservación de los ácidos grasos insaturados de un modo superior a lo habitual, ha sido observada en residuos de cocción de combinaciones de ciertas plantas, y de plantas con carnes, debido a la presencia de antioxidantes vegetales (Malainey, 2007).

En cinco muestras (E266, E267, E268, E270 y E271) se ha detectado presencia de pequeñas cantidades $(0,2$ al $1,8 \%)$ de un isómero trans del ácido oleico (18:1 t) junto con cantidades apreciables de 15:0 (1,2- 5,9\%), 17:0 (2,2-5,8\%) y sus isómeros monoinsaturados 15:1 y 17:1. Resta confirmar si el isómero identificado es el ácido vaccénico (18:1t (n-11) o el ácido elaídico (18:1t (n-9)), lo que permitiría inferir su procedencia. El ácido vaccénico está presente en las grasas de los animales rumiantes (Or-Rashid et al., 2006) mientras que el ácido elaídico es el producto de la isomerización inducida por la luz ultravioleta o las altas temperaturas del ácidos oleico, componente abundante en aceites vegetales.

Además, en cuatro muestras (E266, E268; E270 y E271) se ha identificado ácido linolénico (18:3 (n-6)), cuya presencia en cantidades apreciables estaría circunscrita a ciertas semillas de especies vegetales como la borraja y la grosella negra
(Sergeant et al., 2016). Dado que el 18:3 es un ácido graso muy propenso a oxidación por ser poliinsaturado (Eerkens, 2005), su presencia denota un buen grado de preservación del lípido. Hay experimentos que prueban que cuando una vasija con vegetales ricos en ácidos grasos 18:3 se somete a un tratamiento térmico de cocción, su concentración decae rápidamente (Malainey, 1999). Aún en los casos en que el porcentaje de 18:3 en plantas sea superior al $30 \%$, suele disminuir al $1 \%$ o menos. Por lo que el hecho de encontrarse en cantidades apreciables en el caso en estudio (entre 1,1 a 1,7\%), induce a pensar que estos aceites o tejidos vegetales no eran calentados a temperaturas muy altas, o bien que los poros la preservaron en gran medida. Nuestro equipo de trabajo ha analizado ácidos grasos de varias especies de semillas y plantas patagónicas y no se han detectado cantidades apreciables de este ácido (Barría et al., 2018; Mazzuca et al., 2003; Mazzuca et al., 2001). Para futuras investigaciones se considerará la búsqueda de posibles fuentes que lo contengan.

El análisis cromatográfico de la muestra E270 indicó también la presencia en $0,2 \%$ del ácido poliinsaturado (PUFA) de cadena larga dihomo-gama-linolénico (DGLA) (20:3(n-6)). Cabe observar que la muestra E 270 fue la única que presentó PUFAs superiores a 18 átomos de carbono. La DGLA se halla en general en muy pequeña concentración en plantas y animales y también está presente en ciertos microorganismos (Abu-Gosh et al., 2015). Los típicos PUFA que caracterizan la grasa de peces tales como el eicosapentaenoico (EPA) o el docosahexaenoico (DHA) no han sido identificados. Los mismos raramente 

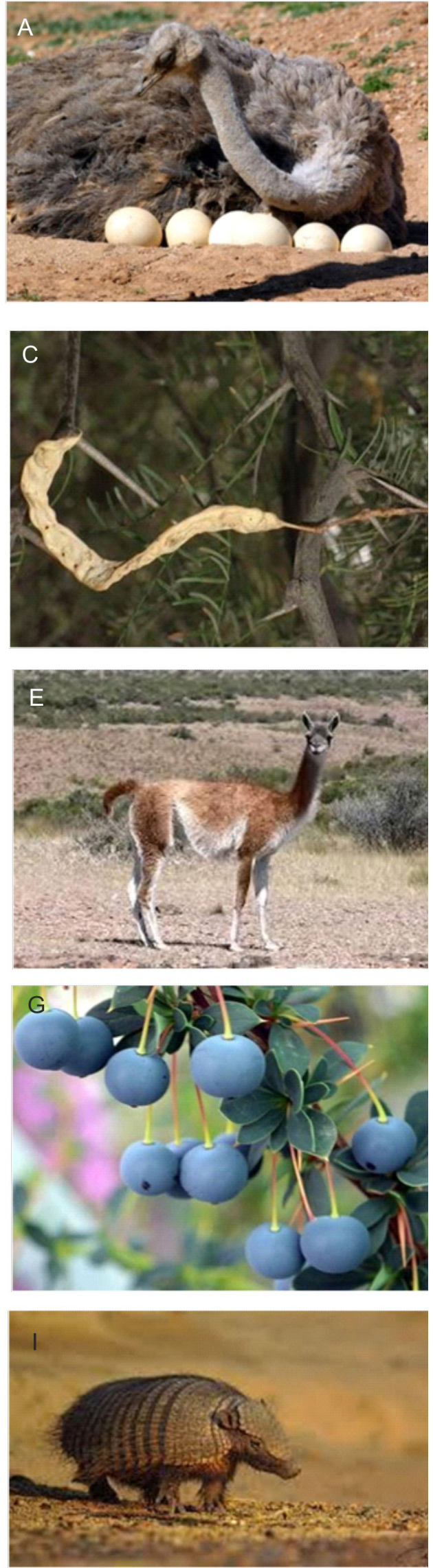
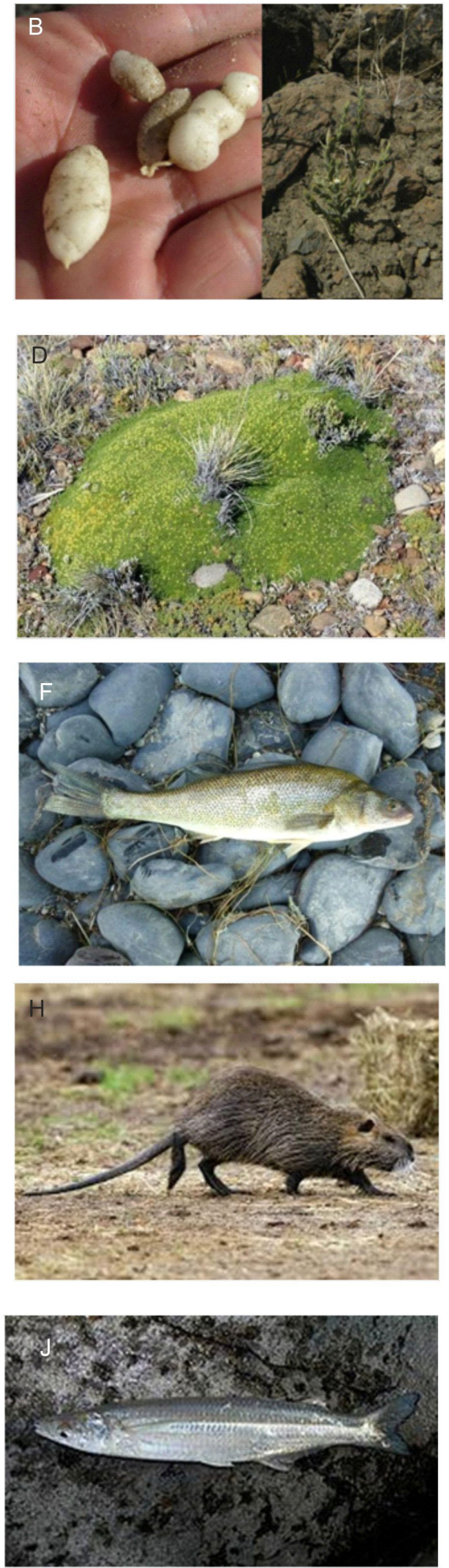

Figura 4. Algunos de los potenciales recursos terrestres y dulceacuícolas del área de estudio:

a) grasa y huevos de choique, Rhea pennata;

b) tubérculos del macachín, Arjona tuberosa;

c) semillas de algarrobo, Prosopis alpataco,

d) raíz de la leña de piedra, Azorella patagónica,

e) guanaco, Lama guanacoide,

f) perca, Perca fluviatilis;

g) frutos del calafate, Berberis microphylla,

h) coipo, Myocastor coypus,

i) piche, Zaedyus pichiy,

j) pejerrey patagónico, Odontesthesmicrolepidotus. 


\begin{tabular}{|c|c|c|c|c|c|c|c|c|c|c|}
\hline AG & E265 & E266 & E267 & E268 & E269 & E270 & E271 & E272 & E273 & E274 \\
\hline 14:00 & 2 & 2,6 & 1,4 & 2,2 & 1,3 & 2,5 & 1,5 & 2,2 & 0,1 & 3,4 \\
\hline 15:00 & 0,9 & 4,4 & 1,2 & 4,6 & 0,8 & 4,4 & 5,9 & 1,6 & 0,6 & 1,9 \\
\hline 16:00 & 41,7 & 31,4 & 38,2 & 29 & 31,4 & 30,1 & 21,2 & 40,9 & 36,2 & 31,8 \\
\hline 17:00 & 0,7 & 2,2 & 2,2 & 3,5 & 0,8 & 5,8 & 3,1 & 1 & 1 & 1,7 \\
\hline 18:00 & 15,6 & 18,2 & 41,6 & 21,7 & 18,7 & 23,9 & 26,8 & 21 & 28 & 28,3 \\
\hline 20:00 & 0,4 & & 0,7 & & & & & & & \\
\hline SFAs & 61,4 & 58,8 & 85,4 & 61 & 53 & 66,7 & 58,6 & 66,8 & 66 & 67 \\
\hline $14: 1 n-5$ & 0,01 & 0,3 & $<0,1$ & 0,45 & $<0,1$ & 0,5 & 0,6 & $<0,1$ & $<0,1$ & $<0,1$ \\
\hline $15: 1 n-5$ & 0,02 & 1,6 & $<0,1$ & 2,03 & $<0,1$ & 1,8 & 2,8 & 0,1 & $<0,1$ & 0,1 \\
\hline $16: 1 n-7 c$ & 6,6 & 3,6 & 1,9 & 2,34 & 3,4 & 2,1 & 2,3 & 4 & 2,6 & 3,5 \\
\hline $17: 1 n-7$ & 0,4 & $<0,1$ & $<0,1$ & $<0,1$ & $<0,1$ & $<0,1$ & 0,1 & 0,1 & 0,1 & 0,5 \\
\hline $18: 1 n-9 c$ & 31,6 & 32,6 & 10,9 & 30,3 & 32,6 & 25,3 & 30,5 & 27,2 & 27,4 & 27,3 \\
\hline $18: 1 \mathrm{n}-9 t$ & & 0,5 & 1,2 & 0,59 & & 0,2 & 1,8 & & & \\
\hline MUFAs & 38,6 & 38,7 & 14 & 35,74 & 36 & 30 & 38,1 & 31,5 & 30,1 & 31,3 \\
\hline $18: 2 n-6 c$ & & 1,4 & 0,6 & 1,95 & 10,9 & 1,9 & 1,6 & 1,6 & 3,9 & 1,6 \\
\hline $18: 3 n-6$ & & 1,1 & & 1,3 & & 1,1 & 1,7 & & & \\
\hline $20: 3 n-6$ & & & & & & 0,2 & & & & \\
\hline PUFAs & & 2,5 & 0,6 & 3,3 & 10,9 & 3,3 & 3,3 & 1,6 & 3,9 & 1,6 \\
\hline UFAs & 38,6 & 41,2 & 14,6 & 39 & 47 & 33,3 & 41,4 & 33,2 & 34 & 32,9 \\
\hline $\mathbf{P} / \mathbf{E}$ & 2,7 & 1,7 & 0,9 & 1,3 & 1,7 & 1,3 & 0,8 & 1,9 & 1,3 & 1,1 \\
\hline
\end{tabular}

Tabla 3. Ácidos grasos identificados en las muestras arqueológicas analizadas. Valores expresados como porcentajes relativos. Referencias: SFAs (saturados), MUFAs (monoinsaturados); PUFAs (poliinsaturados), UFAs (insaturados) (MUFAs+PUFAs), P/E: (índice palmítico/ esteárico).

sobreviven pues son particularmente sensibles a la oxidación (Evershed et al. 2008).

En la muestra E269 -procedente del margen NO del lago Colhue Huapi- se detectaron cantidades elevadas (10\%) de ácido linoleico (18:2(n-6)). Esto es típico de aceites vegetales (Harwood \& Russell, 1984), pero también ha sido identificado en grasa de ñandú en un orden similar $(9 \%)$ (Saadoun \& Cabrera, 2008). Es esperable que si éste ácido proviniera del ñandú como única fuente, su concentración hubiera decaído en el tiempo. En esta muestra además se identificó colesterol, que es una molécula indicadora de grasa animal, infiriéndose que esta muestra presenta rastros de mezcla de grasas de procedencia vegetal y animal. Por último, en las muestras E-272 y E-273 se ha identificado tanto presencia de colesterol como de sitosterol, que es un componente netamente de origen vegetal. Esto permite inferir también el origen mixto del contenido graso presente en estos contenedores cerámicos.

En resumen, el estudio preliminar realizado a las diez muestras procedentes de los sitios arqueológicos del bajo Sarmiento permitió identificar ácidos grasos y esteroles típicos de animales en ocho muestras (E265, E266, E268, E269, E271, E272, E273 y E274). Además en dos de ellas -E272 y E273-, se identificó la presencia de un esterol de origen vegetal, lo que permite inferir que ambas muestras contenían grasas de fuentes mixtas. Dado que la muestra E269 contenía cantidades elevadas de ácidos linoleico, es probable que el residuo sea de origen mixto, y la grasa animal en este caso sea de ñandú. Por último, de confirmarse la identificación del ácido vaccénico es probable que la grasa animal presente en las muestras E266 E267, E268, E270 y E271 sea de rumiante, que en la región patagónica estaría representada por el guanaco (Figura 4).

Al presente, se continúan analizando los resultados de los espectros de masas para identificar aquellos componentes para los cuales no se cuenta con patrones comerciales, así como también la posible presencia de moléculas indicadoras de grasa degradada de organismos acuícolas.

\section{Agradecimientos}

Este trabajo se financió con un subsidio PICT 2015-1932 otorgado al Dr. Moreno y un PUE-CONICET 22920180100012CO concedido al IDEAus (CENPAT-CONICET), instituto que representa a los Dres. Moreno y Schuster. Agradecemos al Sr. Marcos Franco del Laboratorio de Cromatografía y Química Orgánica del CENPAT-CONICET (Puerto Madryn, Chubut) donde se realizaron los análisis cromatográficos de los FAME y los derivados TMS de los esteroles y al Dr. Santi Peralta por la confección de los mapas.

\section{Bibliografía}

Abu Ghosh, S., Pal Nath, D., Markovitch, D., Solovchenko, A., DidiCohen, S., Portugal, I., Khozin-Goldberg, I., Cohen, Z., $\&$ Boussiba, S. (2015). A novel source of dihom $\gamma$ linolenic acid: Possibilities and limitations of DGLA production in the high density cultures of the $\Delta 5$ desaturase mutant microalga Lobosphaera incisa. European Journal of Lipid Science and Technology, 117(6), 760-766.

Barría, M. E., Cretton, M., Gurin, M. C., Arce, M. E., Rost, E. J., \& Mazzuca, M. (2017). Nutrient content of woody species in the Patagonian steppe, Argentina. Boletín de la Sociedad Argentina de Botánica 52 (4): 663-674.

Buonasera, T. (2005). Fatty acid analysis of prehistoric burned 
rocks: a case study from central California. Journal of Archaeological Science, 32(6), 957-965.

Chaile, C., Lantos, I., Maier, M., Cassiodoro, G., \& Tessone A. (2018). Análisis de residuos orgánicos en tecnología cerámica durante el Holoceno tardío en el centro-oeste de Santa Cruz, Argentina. Intersecciones en Antropología, 19: 133-143.

Cordero J. A. \& March R. J. (2013). Análisis de ácidos grasos en fragmentos cerámicos del Noroeste de la Patagonia Argentina por GC y GC-MS. Arqueometría Argentina, estudios pluridisciplinarios (12): 195-220. Universidad Nacional de Lujan.

Eerkens, J. W. (2005). GC-MS analysis and fatty acid ratios of archaeological potsherds from the Western Great Basin of North America. Archaeometry, 47(1), 83-102.

Eerkens, J. W. (2007). Organic residue analysis and the decomposition of fatty acids in ancient potsherds, In Theory and Practice in Archaeological Residue Analysis, Barnard and Eerkens, BAR International Series, 1650, 90.

Evershed R.P., Mottram H.R. Dudd S.N. Charters, Stott A.W., \& Lawrence G.L. (1997). New Criteria for the identification of Animal Fats preserved in Archeological Pottery. Naturwissenschaften 84: 402-406.

Evershed, R. P., Dudd, S. N., Copley, M. S., Berstan, R., Stott, A. W., Mottram, H., Buckley, S. A., \& Crossman, Z. (2002). Chemistry of archaeological animal fats. Accounts of Chemical Research, 35(8), 660-668.

Evershed, R. P; Copley M.S.; Dickinson L.; Hansel F.A. (2008) Experimental evidence for the processing of marine animal products and other commodities containing polyunsaturated fatty acids in pottery vessels. Archaeometry 50: 101-113.

González Díaz, E. F., \& Di Tommaso, I. M. (2014). Paleogeoformas lacustres en el Lago Musters: su relación genética y temporal con aquellas del adyacente lago Colhué Huapí, centro-sur del chubut. Revista de la Asociación Geológica Argentina, 71 (3), 416-426.

Gómez Otero, J. G., Constenla, D., \& Schuster, V. (2014). Análisis de isótopos estables de carbono y nitrógeno y cromatografía gaseosa en cerámica arqueológica del Nordeste de la Provincia del Chubut (Patagonia argentina). Arqueología, 20 (2), 263-284.

Harwood, J. L., \& Russell, N. J. (1984). Lipids in Plants and Microbes, George Allen and Unwin.

Lantos, I., Spangenberg, J. E., Giovannetti, M. A., Ratto, N., \& Maier, M. S. (2015). Maize consumption in pre-Hispanic south-central Andes: chemical and microscopic evidence from organic residues in archaeological pottery from western Tinogasta (Catamarca, Argentina). Journal of Archaeological Science, 55, 83-99.

Maier M.S., de Faria D.L.A., Boschín M.T., Parera S.D., \& Del Castillo Bernal M. F. (2007). Combined use of Vibrational spectroscopy and GC-MS methods in the characterization of archeologicalpastes from Patagonia. Vibrational Spectroscopy 44:182-186.

Malainey, M. E. (1999). The effects of thermal and oxidative degradation on the fatty acid composition of food plants and animals of western Canada: Implications for the identification of archaeological vessel residues. Journal of Archaeological Science 26: 95-103.

Malainey, M. E. (2007). Fatty acid analysis of archaeological residues: procedures and possibilities. BAR International Series, 1650, 77.

Mazzuca, M., \& Balzaretti, V. T. (2003). Fatty acids, sterols and other steroids from seeds of Patagonian Prosopis species. Journal of the Science of Food and Agriculture, 83(10), 1072-1075.

Mazzuca M., Miscoria, S., Rost, E., \& Balzaretti, V. (2004). Ácidos grasos y esteroles en semillas de Ribesrubrum cultivadas en Patagonia. En actas del XXV Congreso Argentino de Química: 426-427. ISBN 950-658-137-1.

Mazzuca M., Miscoria S. A., Rost E., \& Balzaretti, V. T. (2005). Fatty Acids and sterols in sedes from wild species of Berberis in Argentine Patagonia. Anales de la Asociación Química Argentina 93 (4- 6): 241-246. Asociación Química Argentina.

Moreno E. J., Pérez Ruiz, H., \& Ramírez Rozzi, F. (2016). Esquema cronológico y evolución del paisaje en el bajo de Sarmiento (Chubut). F. Mena (Ed.), De mar a mar: 477-485, Ediciones CIEP/ Nire Negro, Santiago de Chile.

Moreno E. J., Pérez Ruiz H., Ramírez Rozzi, F., Reyes, M., Svoboda, A., Peralta González, S., \& Herrera Santana, M. (2015). Primeros resultados de los trabajos arqueológicos en el lago Colhué Huapi (Chubut). Cuadernos del Instituto Nacional de Antropología y Pensamiento Latinoamericano 24 (2): 133-137.

Moreno E. J., \& Svoboda, A. (2013). Explotación de peces y guanacos en el interior de Patagonia central: aportes del sitio Delta del Arroyo Vulcana 1 (Lago Musters, Chubut). Cazadores Recolectores del Cono Sur. Revista de Arqueología 7: 49-68.

Or-Rashid, M. M., Odongo, N. E., \& McBride, B. W. (2007). Fatty acid composition of ruminal bacteria and protozoa, with emphasis on conjugated linoleic acid, vaccenic acid, and odd-chain and branched-chain fatty acids. Journal of Animal Science, 85 (5), 1228-1234.

Patrick, M., de Koning, A. J., \& Smith, A. B. (1985). Gas liquid chromatographic analysis of fatty acids in food residues from ceramics found in the Southwestern Cape, South Africa. Archaeometry, 27 (2), 231-236.

Reyes M. R., \& Svoboda, A. (2016). Un acercamiento a las artes de pesca a partir del análisis de los pesos líticos en el área de los lagos Musters y Colhué Huapi (provincia de Chubut). F. Mena (Ed.), De mar a mar: 496-509, Ediciones CIEP/ Nire Negro, Santiago de Chile.

Saadoun, A., \& Cabrera, M. C. (2008). A review of the nutritional content and technological parameters of indigenous sources of meat in South America. Meat Science, 80 (3), 570-581.

Sergeant, S., Rahbar, E., \& Chilton, F. H. (2016). Gammalinolenic acid, dihommo-gamma linolenic, eicosanoids and inflammatory processes. European Journal of Pharmacology, 
$785,77-86$.

Schuster V. 2014. La organización tecnológica de la cerámica de cazadores recolectores. Costa norte de la provincia del Chubut (Patagonia argentina). Relaciones de la Sociedad Argentina de Antropología XXXIX (1): 353-366.

Stoessel, L., Martínez, G., \& Constenla, D. (2015). Análisis preliminar de ácidos grasos recuperados de cerámicas arqueológicas del curso del Río Colorado (Norpatagonia Oriental): Aportes para la subsistencia de grupos cazadoresrecolectores. Magallania 43(1), 231-249.

Svoboda A., \& Moreno E. J. (2018). Peces y coipos: zooarqueología del sitio Valle Hermoso 4 (Lago Colhué Huapi, Chubut). Revista del Museo de Antropología 1 (1): 85-98. Universidad Nacional de Córdoba.

Svoboda A. (2019). Ictioarqueología del sitio Boliche Jeréz 3 (Lago Colhué Huapi, Chubut, Argentina): implicaciones para la subsistencia de cazadores-recolectores del Holoceno tardío. Revista de Antropología del Museo de Entre Ríos 5 (1): 54-67. 
